\title{
Editorial
}

\section{Fungus-Fungus Interactions}

\author{
Susanne Zeilinger-Migsich ${ }^{1, *}$ and Prasun K. Mukherjee ${ }^{2}$ \\ ${ }^{1}$ Research Division Biotechnology and Microbiology Institute of Chemical Engineering (E166-5) Vienna University of \\ Technology, Gumpendorferstraße 1a, A-1060 Wien, Austria \\ ${ }^{2}$ Nuclear Agriculture and Biotechnology Division, Bhabha Atomic Research Centre, Trombay, Mumbai 400085, India
}

1.5 million fungal species are estimated to exist on our planet which are assumed to account for at least $25 \%$ of the global biomass. Fungi inhabit a broad range of environmental niches and, due to their nutritional versatility, perform many essential processes like nutrient recycling and decomposition in global ecosystems. In their natural environment, fungi closely interact with other organisms such as other fungi, bacteria, plants and animals.

Both intra- as well as inter-specific fungal interactions may be mediated upon contact or even at a distance and include communication between the interaction partners. For communication, fungi use a "chemical language" and these signaling molecules result in responses, i.e. a certain behavior, in the recipient leading to e.g. mating, alterations in growth and development, and pathogenicity. In many cases, the specificity and functions of these interactions are not well understood and the chemoattractive mechanisms involved are largely unknown.

As do other organisms, fungi are also able to distinguish between "self" and "non-self". While hyphal networks can be formed by fusion between colonies that are genetically identical, hyphal fusion between non-self colonies results in hyphal interference and programmed cell death. In this special issue, these aspects including recognition and avoidance mechanisms are discussed by Lichius and Lord.

*Address correspondence to this author at the Research Division Biotechnology and Microbiology Institute of Chemical Engineering (E1665) Vienna University of Technology, Gumpendorferstraße 1a, A-1060 Wien, Austria; Tel: +43 158801 166560; Fax: +43 158801 17299;

E-mail: szeiling@mail.tuwien.ac.at
In their natural environment, fungi have to compete with other organisms and among each other for resources such as nutrients and space. Competition can occur by e.g. the secretion of secondary metabolites or by direct interaction such as overgrowth and mycoparasitism. Mycoparasitism, where one fungus attacks and invades another, significantly contributes to suppression of pathogen population in nature, and is applied in the biological control of plant diseases. The best studied mycoparasites belong to the genus Trichoderma, antagonistic fungi which are used as microbial biological control agents in agriculture. The review article of Sawant gives an overview on the interactions of Trichoderma with foliar pathogens and Woo et al. in their article address the application of Trichoderma-based products in agriculture. In addition to organismic biocontrol agents, substances derived thereof can also be applied. Some secondary metabolites have been found to play important roles in the biocontrol mechanisms of beneficial fungi by not only inhibiting or killing competitors but also by directly affecting the plant. In this special issue, Vinale et al. review the role of secondary metabolites in beneficial fungus-fungus interactions.

Understanding the basic mechanisms is essential to improve any system, and biocontrol is no exception. Trichoderma spp., being widely applied, also attracted significant attention as model systems for understanding fungus-fungus interactions at the molecular level. Sarma and co-authors have touched upon this topic illustrating the mode of action of these biocontrol fungi.

With more and more consumers being concerned about the negative effects of chemicals applied in agriculture, the pest management practice has to tilt towards biological control and we hope the publication of this special issue will stimulate our interests in this area.

(C) Zeilinger-Migsich and Mukherjee; Licensee Bentham Open.

This is an open access article licensed under the terms of the Creative Commons Attribution Non-Commercial License (http://creativecommons.org/ licenses/by-nc/3.0/), which permits unrestricted, non-commercial use, distribution and reproduction in any medium, provided the work is properly cited. 